\title{
Observations of the Near-Infrared Extragalactic Background Light
}

\author{
Toshio Matsumoto \\ Institute of Space and Astronautical Science, Kanagawa 229-8510, Japan
}

\begin{abstract}
We searched for the near infrared extragalactic background light (IREBL) in data from the Near Infrared Spectrometer (NIRS) on the Infrared Telescope in Space (IRTS). After subtracting the contribution of faint stars and a modeled zodiacal component, a significant isotropic emission is detected whose in-band flux amounts to $\sim 30 \mathrm{nWm}^{-2} \mathrm{sr}^{-1}$. This brightness is consistent with upper limits of COBE/DIRBE, but is significantly brighter than the integrated light of faint galaxies. The star subtraction analyses from DIRBE data show essentially the same results apart from the uncertainty in the model of the zodiacal light. A significant fluctuation of the sky brightness was also detected. A 2-point correlation analysis indicates that the fluctuations have a characteristic spatial structure of $100 \sim 200 \mathrm{arcmin}$. This could be an indication of the large scale structure at high redshift. Combined with the far infrared and submillimeter EBL, the total energy flux amounts to $50 \sim 80 \mathrm{nWm}^{-2} \mathrm{sr}^{-1}$ which is so bright that unknown energy sources at high redshifts are required.
\end{abstract}

\section{Introduction}

The infrared extragalactic background light (IREBL) provides an important clue to the understanding of the early universe and the evolution of galaxies. In the near infrared, redshifted star light from high $\mathrm{z}$ galaxies constitutes the background, while dust emission produces the far infrared and submillimeter background. Both emission components are thought to be important parameters to understand energy generation during the galaxy formation era. It is thought that the near infrared $(1 \sim 5 \mu \mathrm{m})$, and far infrared/submillimeter $(100 \sim 300$ $\mu \mathrm{m}$ ) wavelength bands are useful windows to detect the IREBL since sky brightness is very low there. Many efforts have been devoted to detect the EBL from the ground but these were not successful due to bright atmospheric and instrumental emission. Recent space observations from COBE and IRTS first enabled a qualitative analysis of the IREBL.

Puget et al. (1996) and Fixsen et al. (1998) reported a significant detection of the submillimeter EBL from the COBE/FIRAS data. Furthermore, Hauser et al. (1998) claimed the detection of the EBL at 120 and $240 \mu \mathrm{m}$ from the COBE/DIRBE data, but obtained only upper limits in the near infrared wavelength bands. The difficulty with DIRBE observations of the near infrared EBL 
is mainly the relatively large beam size of $0.7^{\circ}$ that resulted in serious confusion from unresolved foreground stars.

In this paper, we present the detection of the near infrared EBL with the data of IRTS/NIRS and discuss its cosmological implications.

\section{Observations and Selection of the Data}

The Near InfraRed Spectrometer (NIRS) is one of the focal plane instruments of the InfraRed Telescope in Space (IRTS), and was optimized to observe the spectrum of the diffuse background (Noda et al. 1994). The NIRS covers the wavelength range from $1.4 \mu \mathrm{m}$ to $4.0 \mu \mathrm{m}$ with a spectral resolution of $0.13 \mu \mathrm{m}$. The beam size is 8 arcmin square, which is considerably smaller than that of DIRBE.

IRTS was one of the mission experiments on the small space platform, SFU, that was launched on March 18, 1995. The IRTS observations lasted for about 30 days, and $7 \%$ of the sky was surveyed (Murakami et al. 1996). During the IRTS mission period, many bright stars were observed, and absolute calibration and measurement of the beam pattern was satisfactorily attained with the flight data (Noda et al. 1996).

In the initial phase of observations, NIRS observed the environmental $\mathrm{OH}$ emission in the southern hemisphere, which had a peak at about $2.8 \mu \mathrm{m}$. Passages through the South Atlantic Anomaly (SAA) and the incidence of lunar radiation into the telescope tube also seriously affected the detectors. To avoid these effects, only the data for the last 5 days, those of orbits that did not pass through the SAA and that did not suffer from lunar radiation, and those of the northern sky were used for this analysis.

Further, in order to reduce the contribution from faint stars, the sky at high galactic latitudes $\left(b>40^{\circ}\right)$ was chosen. The highest galactic latitude is $58^{\circ}$, and the ecliptic latitude ranges from $12^{\circ}$ to $71^{\circ}$ in the selected data.

The data were selected from $5 \mathrm{sec}$ integrations during which no distinguishable stars and no hits of cosmic rays were detected in any wavelength band. This procedure resulted in an effective beam size of 8 arcmin $\times 20$ arcmin due to the scanning effect. Finally, full spectra of the sky at 1010 positions were obtained.

Figure 1 shows the averaged spectrum of the sky at high ecliptic latitude $\left(\beta>70^{\circ}\right)$ in which the data of the DIRBE darkest sky (Hauser 1995) are also presented. Figure 1 implies that both data are fairly consistent despite the difference in the observed regions and beam size.

\section{Subtraction of Foreground Emission}

\subsection{Contribution of Faint Stars}

One of the superior characteristics of the NIRS observations is that fainter stars can be identified and subtracted due to the reduced confusion owing to the smaller beam size than that of COBE/DIRBE.

The first step in subtracting the stellar component is to obtain the number density of stars $(\log N / \log S$ relation). Fortunately, NIRS detected several tens of thousands of stars during the IRTS mission period, and a complete catalog is 


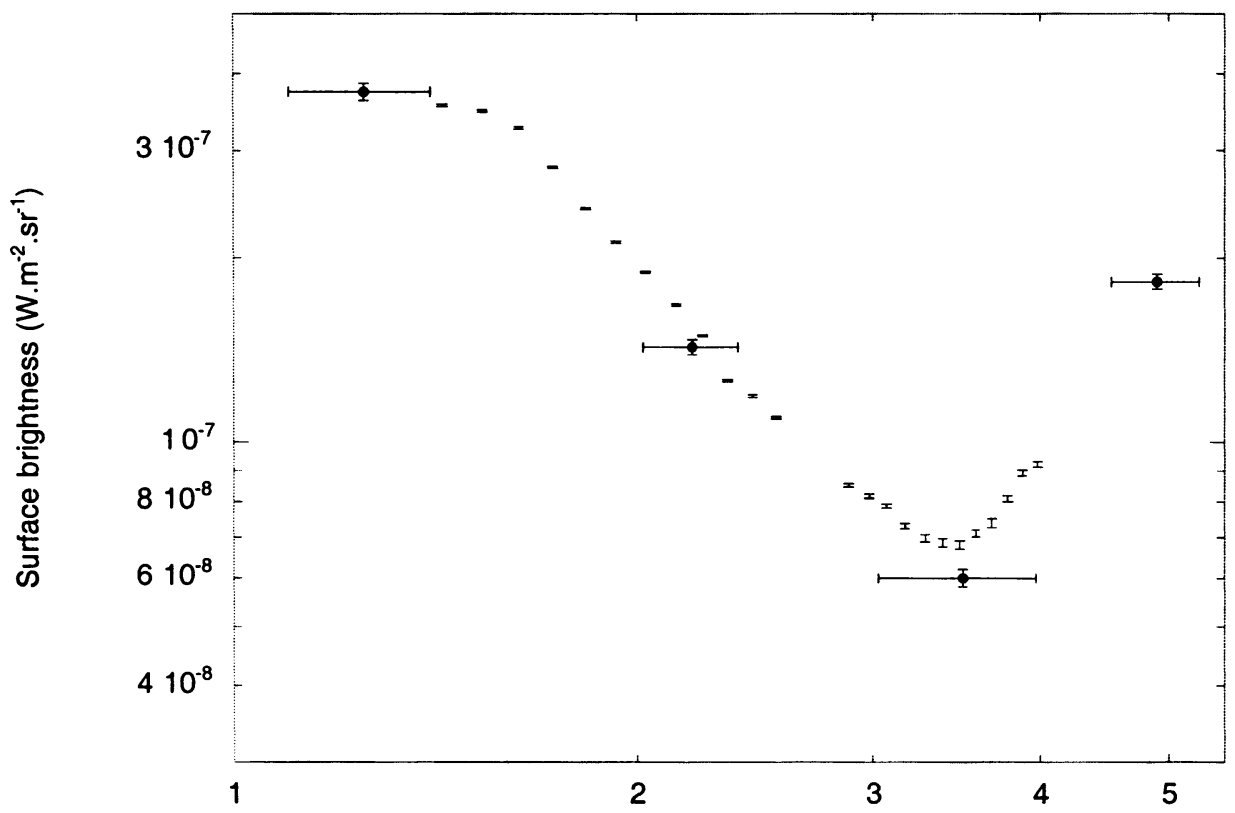

Wavelength $(\mu \mathrm{m})$

Figure 1. The averaged spectrum of the sky at high ecliptic latitude $\left(\beta>70^{\circ}\right)$. Vertical bars indicate the errors, in which systematic errors of calibration are dominant. Filled circles indicate the DIRBE darkest sky for each band (Hauser 1995). 
being prepared. Although this catalog is not so deep (completeness at $2.24 \mu \mathrm{m}$ is $7.5 \mathrm{mag}$ ), we constructed the $\log N / \log S$ relation to 20th magnitude in all wavelength bands based on a model Galaxy (Cohen 1997). The uncertainty in the $\log N / \log S$ relation is $\sim 10 \%$.

The second step is to find the contribution of stars that are too faint to be resolved in the acquired data. We define the cut-off magnitudes as the brightest stars in the acquired data which are obtained with stellar fluxes that correspond to the noise with the same spatial frequency as the beam pattern. The cut-off magnitude at $2.24 \mu \mathrm{m}$ thus obtained is $10.5 \mathrm{mag}$. Uncertainties are $\pm 0.5 \mathrm{mag}$ nitudes for all wavelength bands.

With these $\log N / \log S$ relations and cut-off magnitudes, we calculated the surface brightness due to faint stars at $b=42^{\circ}, 45^{\circ}$, and $48^{\circ}$ along the scan path. We regarded these three data sets as representative values for the sky regions at $40^{\circ}<b<42.5^{\circ}, 42.5^{\circ}<b<47.5^{\circ}$, and $47.5^{\circ}<b$, respectively. The difference of the brightness in these three positions is about $10 \%$, and errors are 5 to $10 \%$ depending on the wavelength band. The contribution of faint stars in the $2.24 \mu \mathrm{m}$ band is about $10 \%$ of the observed sky brightness at high ecliptic latitude.

The integrated light of the faint stars for each wavelength band was subtracted from the observed sky brightness. This "star subtracted data" is used in the subsequent analysis.

\subsection{Subtraction of the Zodiacal Component}

Recently Kelsall et al. (1998) constructed a physical model for interplanetary dust (IPD) using the seasonal variation of the zodiacal light and emission. Based on this physical model we calculated the brightness of the zodiacal component corresponding to our wavelength bands and observed points and subtracted this model brightness from the "star subtracted data".

Figure 2 shows the ecliptic latitude dependence of the star subtracted brightness, model zodiacal component, and residual emission after subtracting the stars and zodiacal component at $2.24 \mu \mathrm{m}$. Other wavelength bands show essentially the same features. The model brightness of the zodiacal component is calculated for all observed positions but the results show no dependence on ecliptic longitude. Therefore, the model brightness in Figure 2 appears as a continuous curve.

Figure 2 clearly shows that there remains a fairly isotropic residual emission after subtracting the faint stars and zodiacal component. Figure 3 indicates the correlation between "star subtracted data" and model brightness at $2.24 \mu \mathrm{m}$. The correlation is excellent for all bands, but the slopes in the long wavelength bands are a little steeper than 1.0, which implies that the model is not perfect at long wavelengths. To attain a minor correction to the model, we made linear fits for the correlation between the model brightness and star subtracted brightness leaving the slopes to be free parameters. The isotropic emission is defined as the brightness where the model brightness is zero. Errors are estimated by taking into account fitting errors, uncertainties in the cut-off magnitudes and models of the Galaxy and IPD, and systematic errors of the calibration. Fitting errors are small compared with other errors, and the major source of the errors is the uncertainty in the IPD model that is estimated by interpolating the uncertainties 


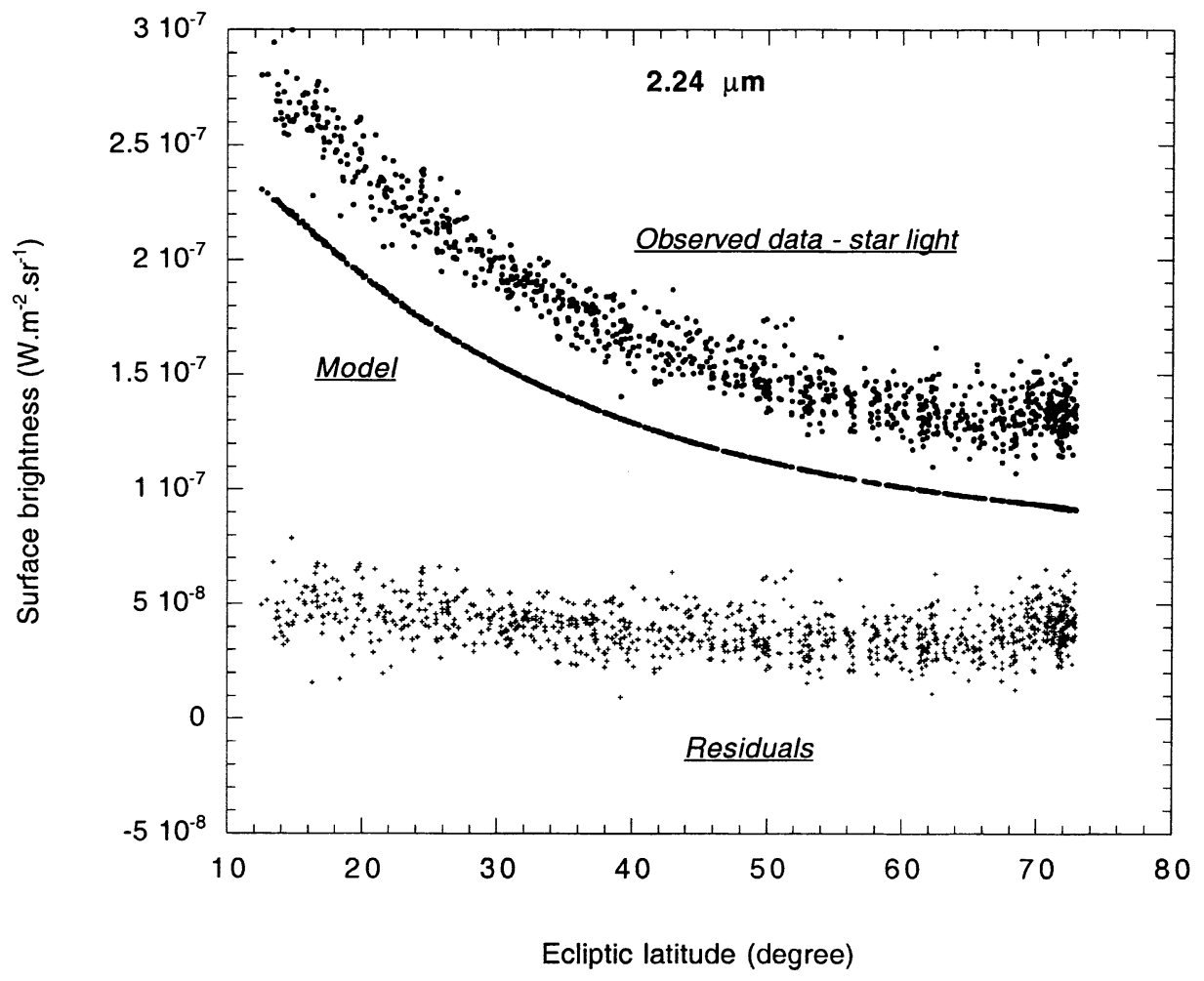

Figure 2. The dependence of data at $2.24 \mu \mathrm{m}$ on ecliptic latitude. Dots, solid lines, and crosses, respectively, indicate star subtracted brightness, IPD model, and residual brightness after subtracting the stars and zodiacal component from the observed sky brightness. 


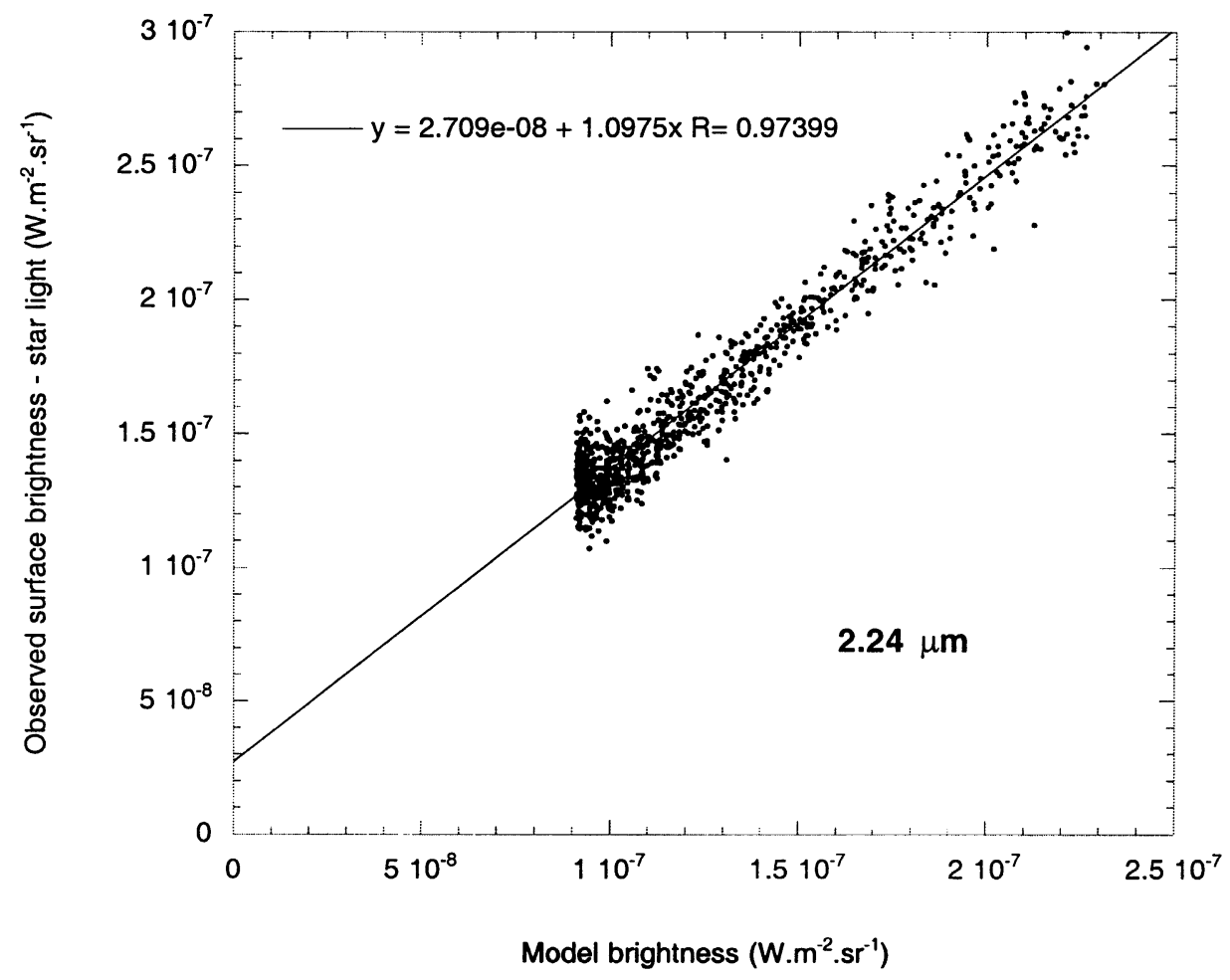

Figure 3. Correlation diagram between star subtracted brightness and the model brightness at $2.24 \mu \mathrm{m}$.

in the original model brightness $\left(15,6,2.1\right.$, and $5.9 \mathrm{nWm}^{-2} \mathrm{sr}^{-1}$, respectively, for the J, K, L and M bands; Kelsall et al. 1998).

Figure 4 shows the spectrum of the observed isotropic emission compared with other observations and theory. The isotropic emission obtained is consistent with DIRBE upper limits (Hauser et al. 1998) but considerably higher than the integrated light of galaxies compiled by Totani et al. (2000). It must be noted that the recent theory by Totani and Yoshii (2000) is fairly consistent with the integrated light of observed galaxies. The spectrum of the isotropic emission is stellar, and no clear band feature is found. In-band energy of the isotropic emission amounts to $\sim 30 \mathrm{nWm}^{-2} \mathrm{sr}^{-1}$.

\section{Comparison with DIRBE Minus Stars}

Matsumoto et al. (2000) tried to search for the EBL by subtracting the stellar contribution from DIRBE data. They carried out star counts in the $\mathrm{J}$ band with the Kiso Schmidt Telescope. They observed one degree square towards the DIRBE dark spot with completeness down to 14 mag., and detected 334 stars. They could subtract the sky brightness due to detected stars directly from the DIRBE data. As for the unresolved faint stars, they applied Cohen's sky model 


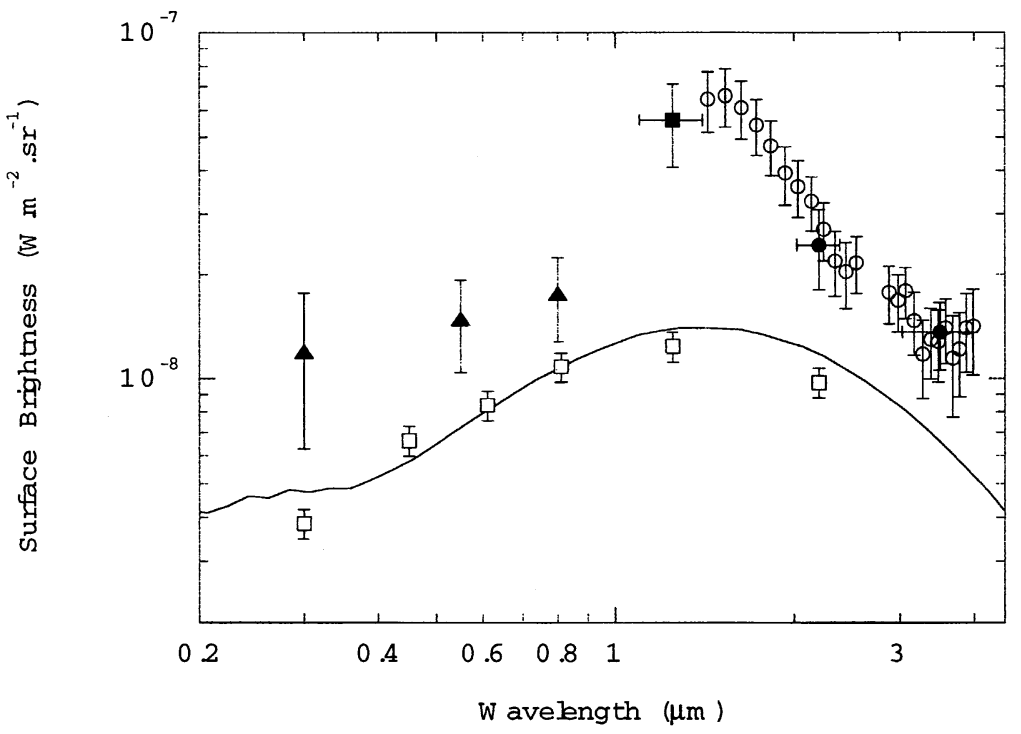

Figure 4. Spectrum of the observed isotropic emission. NIRS data are shown by open circles. Filled circles and the filled square show the detection of EBL reported, respectively, by Gorjian, Wright, \& Chary (2000) and Matsumoto et al. (2000) with DIRBE-minus-stars analyses. Filled triangles indicate the recent detection of the EBL by Bernstein, Freedman, \& Madore (2000). Open squares represent the integrated light of galaxies compiled by Totani et al. (2000). The recent SUBARU data at the $\mathrm{J}$ and $\mathrm{K}$ bands are included, and correction for the diffuse extended emission is attained for all bands. The solid line shows the model prediction of the NIR EBL by Totani \& Yoshii (2000). 
(Cohen 1997)). Since the contribution of faint unresolved stars is only $12 \%$ of the total star light, the ambiguity of the model is not serious. They determined a significant residual emission which amounts to $60.1 \pm 15 \mathrm{nWm}^{-2} \mathrm{sr}^{-1}$ in the $\mathrm{J}$ band which is fairly consistent with the IRTS/NIRS results (see Figure 4).

Gorjian, Wright, \& Chary (2000) published similar work and Wright (2000) extended this work using 2MASS data. Wright (2000) obtained an EBL brightness of $27.8 \pm 14.5 \mathrm{nWm}^{-2} \mathrm{sr}^{-1}$ and $19.9 \pm 5.3 \mathrm{nWm}^{-2} \mathrm{sr}^{-1}$, respectively, for the $\mathrm{J}$ and $\mathrm{K}$ bands. These values are somewhat lower than the IRTS/NIRS results, due to differences in the zodiacal light model. If we apply Kelsall's model to Wright's values, they are modified to $60.8 \pm 14.5 \mathrm{nWm}^{-2} \mathrm{sr}^{-1}$ and $28.2 \pm 5.5$ $\mathrm{nWm}^{-2} \mathrm{sr}^{-1}$ for the $\mathrm{J}$ and $\mathrm{K}$ band, respectively, which are fairly consistent with the Kiso star counts and the IRTS/NIRS results.

Table 1 gives a summary of results in which the difference due to the model employed is also shown. COBE/DIRBE and IRTS/NIRS render the same results for the sky brightness; however, a difference in the chosen model causes a serious difference in the estimation of the EBL, especially for the short wavelength bands. In Figure 4, the results based on Kelsall's model (Kelsall et al. 1998) are plotted for consistency.

Table 1. Summary of observations of DIRBE minus stars. The units are $\mathrm{nWm}^{-2} \mathrm{sr}^{-1}$. Numbers in parentheses indicate the corrected brightness with Kelsall's model

\begin{tabular}{ccc}
\hline \hline Authors & J band & K band \\
\hline Gorjian et al. (2000) & & $16.2 \pm 6.4(24.5 \pm 6.6)$ \\
Wright (2000) & $27.8 \pm 14.5(60.8 \pm 14.5)$ & $19.9 \pm 5.3(28.2 \pm 5.5)$ \\
Matsumoto et al. 2000 & $60.1 \pm 15$ & \\
IRTS/NIRS & & $27.5 \pm 5$ at $2.24 \mu \mathrm{m}$ \\
\hline
\end{tabular}

\section{Fluctuation Analysis}

Fluctuations of the extragalactic background light are another important clue in the study of the formation and evolution of galaxies. The effective beam size of 8 arcmin $\times 20$ arcmin is an adequate scale to investigate the clustering of galaxies at high redshift (Kashlinsky \& Odenwald 2000).

For the fluctuation analysis with the NIRS data, we constructed the "fluctuation data set" by extracting the fluctuating component from the star subtracted data. We adopted a baseline obtained by fitting the ecliptic latitude dependence (Figure 2) to third order polynomials to avoid the effects of incompleteness of the IPD model on the large scale structure. The standard deviations are obtained assuming a gaussian distribution which are shown by filled circles in Figure 5.

Fluctuations due to faint stars are estimated by a Monte-Carlo simulation with cut-off magnitudes and a model Galaxy where a random distribution is assumed. The read out noises were obtained from the fluctuations of signals when the shutter was closed. It was found that observed fluctuations consist of read out noise on the longer wavelength side, but there remains an unknown fluctuation component on the short wavelength side. In Figure 5, open circles indicate 


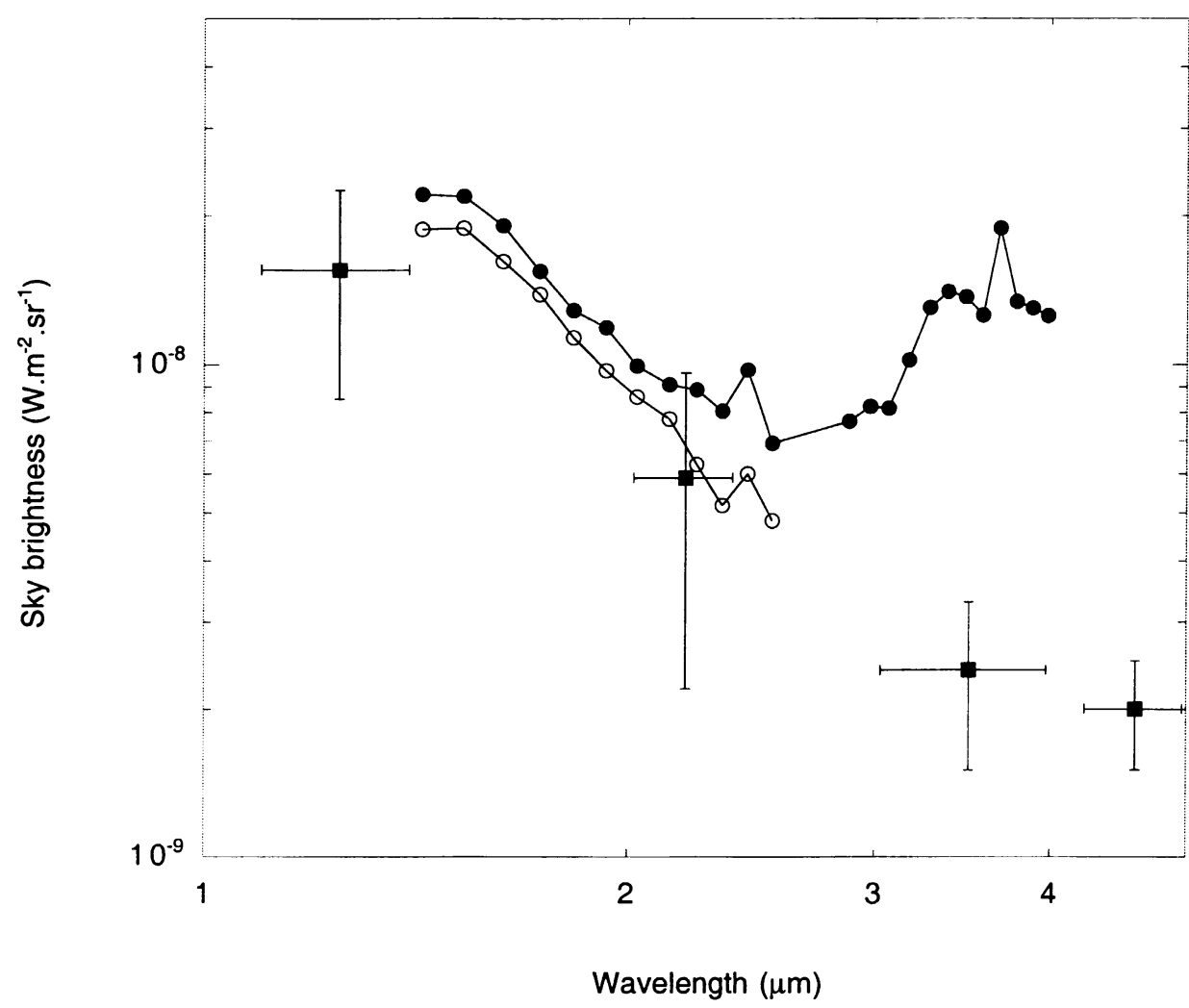

Figure 5. Spectrum of rms fluctuations observed by the IRTS shown by filled circles. Open circles indicate the excess fluctuation after subtracting read out noise and stellar fluctuations. Squares are rms fluctuations obtained for the DIRBE data by Kashlinsky \& Odenwald (2000).

the residual fluctuations that were obtained by subtracting the fluctuation due to stars and read out noise in quadrature.

We found that there exists a clear correlation between wavelength bands for the "fluctuation data set". The correlation analysis for the fluctuation data set indicates that the color of the fluctuating component is very similar to the spectrum of the isotropic emission in Figure 4. This implies that the observed excess fluctuations have the same origin as the isotropic emission, in other words, the isotropic emission fluctuates keeping the same spectral shape.

The zodiacal component can not explain this fluctuation. The fluctuation of zodiacal emission has not been detected in the mid infrared region and is less than $1 \%$ for various beam sizes (IRAS: Vrtilek \& Hauser 1995; COBE: Kelsall et al. 1998; ISO: Abraham et al. 1998). The upper limits thus obtained are much smaller than the observed fluctuating component, that is, $1 / 4$ of the isotropic emission and $1 / 20$ of the total sky brightness. Since it is unlikely that the scattering component has a much larger fluctuation than that of the thermal part, it is difficult to attribute the excess fluctuations to zodiacal light. The fact that 
the fluctuation of the residual emission in Figure 2 has no dependence on ecliptic latitude is further evidence that the observed fluctuation is not interplanetary in origin.

The contribution of faint stars to the fluctuation is not as clear since the spatial distribution of stars may not be random. Furthermore, it is difficult to confirm this, since an infrared star catalog deep enough for this purpose is not yet available. However, in order to interpret the observed excess fluctuation as due to faint stars, the fluctuations due to faint stars would have to be three to four times that of the simulation. Considering that the contribution of the faint stars is overestimated in our analysis, it is hard to explain the observed excess fluctuation with faint stars.

In Figure 5, the recent fluctuation analysis of the DIRBE data by Kashlinsky \& Odenwald (2000) is also shown by squares. Although the error bars are fairly large, good agreement is found between the two independent analyses. Figure 5 implies that the origin of the observed excess fluctuation is not stellar, and that the angular scale of the fluctuation could be comparable or larger than the DIRBE beam $\left(0.7^{\circ}\right)$.

In order to investigate the nature of the fluctuations, a two point correlation analysis is crucial. To obtain a definite result, we defined the "wide band brightness" by integrating the brightness of the short wavelength bands from $1.43 \mu \mathrm{m}$ to $2.14 \mu \mathrm{m}$. The rms fluctuation of the "wide band brightness" was found to be $6.6 \mathrm{nWm}^{-2} \mathrm{sr}^{-1}$ of which read out noise amounts to $1.5 \mathrm{nWm}^{-2} \mathrm{sr}^{-1}$, and the fluctuation due to faint stars is estimated to be $2.1 \mathrm{nWm}^{-2} \mathrm{sr}^{-1}$.

The two point correlation function is defined as $C(\theta)=\langle\delta F(x+\theta) \delta F(x)>$, where $\delta F(x)$ is the fluctuating component of the "wide band brightness", $\theta$ is the angular distance in arcminutes between two points, and $x$ are the coordinates of the observed points. The left panel of Figure 6 shows the two point correlation function thus obtained. Error bars are indicated for selected points. The increase at the left end could be due to a proximity effect since some data points overlap on the scale of the beam size. Correlations at large angular scales $(\theta>800$ arcmin $)$ are not adopted since large scale structure is seriously affected by uncertainties in the base line used to extract the "fluctuating component". On the other hand, the structure at $100 \sim 200$ arcmin is statistically significant. Simulations with random data do not show fluctuations with this scale, that is, sampling effects are not the cause.

The two point correlation function can be converted into a power spectrum, $P(q)$, where $q$ is a wavenumber in units of $\operatorname{arcmin}^{-1}$. Here, we adopted a one dimensional Fourier transformation with $\theta$, since data points lie on the narrow belt and the beam pattern is elongated along the scan path. The result is shown in the right panel of Fig.6 where $(q P(q) / 2 \pi)^{1 / 2}$ is plotted as a function of $1 / q$. As expected, a clear hump at $100 \sim 200$ arcmin is found.

\section{Discussion}

In Figure 7, recent results of the submillimeter and far infrared EBL are shown together with the NIR EBL. Puget et al. (1996) first reported the detection of the EBL in the submillimeter region with DIRBE data, which was later confirmed by Fixsen et al. (1998). In the far infrared region, Hauser et al. (1998) reported 

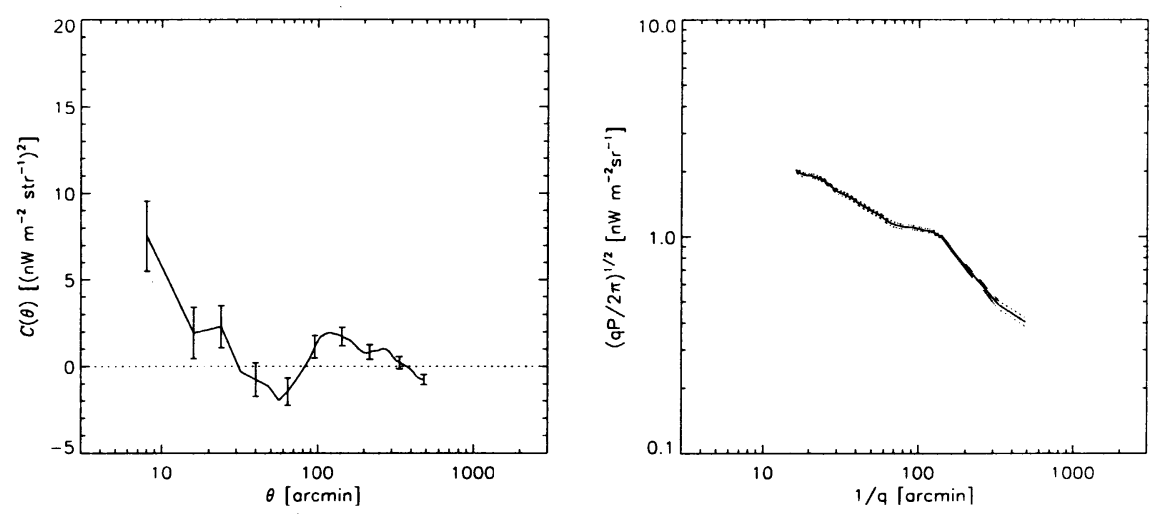

Figure 6. Left panel: Two point correlation function shown as a function of angular distance $\theta$. Right panel: Power spectrum $(q P(q) / 2 \pi)^{1 / 2}$ shown as a function of $1 / q$.

a significant detection of the EBL at 140 and $240 \mu \mathrm{m}$, and upper limits at 60 and $100 \mu \mathrm{m}$. Lagache et al. (2000) carried out a careful analysis and obtained the EBL at 100, 140 and $240 \mu \mathrm{m}$, consistent with Hauser et al. (1998). Recently, Finkbeiner et al. (2000) introduced a new analysis to subtract the zodiacal component, and reported the detection of the EBL even at $60 \mu \mathrm{m}$.

The observed FIR and submillimeter EBL should be compared with the integrated light of point sources. At $850 \mu \mathrm{m}$, deep surveys with SCUBA have been carried out. Blain et al. (1999) made the deepest survey, using the lensing effect, and reported that SCUBA sources can explain the submillimeter background. In the far infrared region, ISO surveys are the sole observations available. The detection limits of the ISO surveys were not deep enough to estimate the contribution of the point sources to the EBL. However, Matsuhara et al. (2000) made a fluctuation analysis with a deep survey toward the Lockman hole where the column density of neutral hydrogen is a minimum. They first detected fluctuations due to faint galaxies and obtained galaxy number counts down to a level a few times lower than the detection limits of ISO. They found that strong evolution is required to explain the detected fluctuations. The background due to faint galaxies in their model, however, is still significantly lower than the observed EBL.

The most important issue of the recent observations of the EBL is energetics. Combining the NIR and FIR/submillimeter EBL, the total energy flux is estimated to be $50 \sim 80 \mathrm{nWm}^{-2} \mathrm{sr}^{-1}$. Assuming a single star burst at a redshift $z_{f}$, the following simple expression is obtained:

$$
\text { Total energy flux } \sim 10\left(\frac{h^{2} \Omega_{B}}{0.02}\right)\left(\frac{\Delta X}{0.01}\right)\left(\frac{5}{1+z_{f}}\right) \mathrm{nWm}^{-2} \mathrm{sr}^{-1}
$$

where $\Omega_{B}$ and $\Delta X$, respectively, are the baryon density parameter and conversion factor from hydrogen to helium. Since $\Omega_{B}$ and $\Delta X$ are seriously constrained by nuclear synthesis and metal abundance, it does not appear easy to explain 


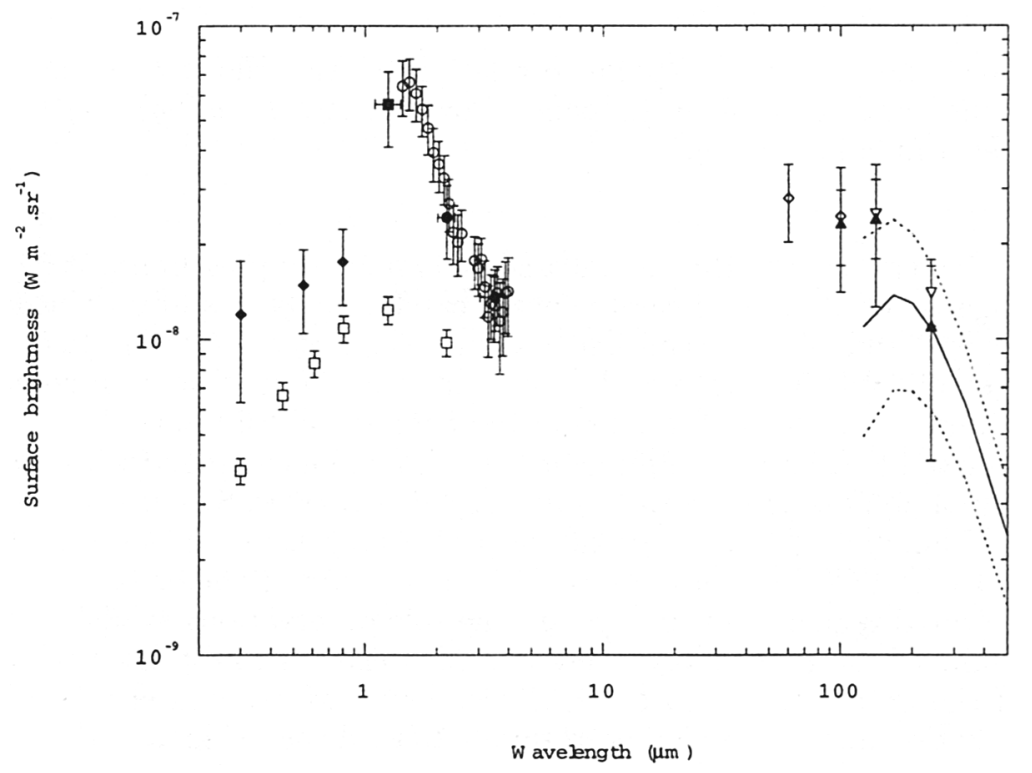

Figure 7. Summary of recent results on the IREBL. Filled circles and filled squares show the detection of EBL reported by Gorjian et al. (2000) and Matsumoto et al. (2000) with DIRBE minus stars analyses, respectively. Filled diamonds indicate the recent detection of EBL by Bernstein et al. (2000). Open squares represent integrated light of galaxies compiled by Totani et al. (2000). Open triangles, filled triangles, and open diamonds indicate the results by Hauser et al. (1998), Lagache et al. (2000), Fixsen at al. (1998), respectively. 
the observed EBL by known objects. The observed IREBL requires new energy sources and/or hidden objects.

Another important piece of observational evidence is a significant detection of fluctuations in the NIR EBL. The observed rms fluctuation level is fairly large ( 1/4 of the isotropic emission), but is marginally consistent with the model by Kashlinsky \& Odenwald (2000). Furthermore, the detected two point correlation function and PSD show typical angular scales of $100 \sim 200$ arcmin. Since the co-moving scale of $10 \mathrm{Mpc}$ at a redshift $\sim 10$ corresponds to an angular scale of $\sim 1^{\circ}$, the observed spatial structure could be evidence of the large scale structure at high redshift.

Considering that the fluctuating component has a similar spectral shape to that of the isotropic emission, these observational results suggest that the observed EBL originates in a short period at high redshift. Population III objects or MACHO progenitors could be candidates for these energy sources (Madau and Pozzetti 2000)

Another interesting topic of the bright EBL is intergalactic absorption of $\mathrm{TeV} \gamma$ rays (inverse process of pair creation). The NIR photons cause an absorption peak around $1 \mathrm{TeV}$, while FIR photons are effective in the higher energy range. The NIR EBL detected by IRTS/NIRS gives an optical depth $\tau \sim 1$ for the well known $\mathrm{TeV} \gamma$-ray blazars Mrk421 and Mrk 501. The IREBL is now very important for the study of the emission mechanism of $\mathrm{TeV} \gamma$-rays. Details of this topic will be found in a separate paper (Tanihata, Takahashi, \& Matsumoto 2000).

Acknowledgments. The author thanks IRTS members for their encouragement and useful discussions with them. He also thanks Dr. Totani for providing SUBARU data.

\section{References}

Abraham, P. et al 1998, in ESA SP-427, The Universe as Seen by ISO, ed. P. Cox \& M. Kessler, 145

Bernstein, R. A., Freedman, W. L., \& Madore, B. F. 2000, ApJ, submitted

Blain, A. W., Kneib, J. -P, Ivison, R. J., \& Smail, I. 1999, ApJ, 512, L87

Cohen, M. 1997, in ASP Conf. Series Vol. 124, Diffuse Infrared Radiation and the IRTS, ed. H. Okuda, T. Matsumoto \& T. Roellig (San Francisco: ASP), 61

Finkbeiner, D. P., Davis, M., \& Schlegel, D. J. 2000, ApJ, 544, 81

Fixsen, D. J. et al. 1998, ApJ, 508, 123

Gorjian, V., Wright, E. L., \& Chary, R. R. 2000, ApJ, 536, 550

Hauser, M. G. 1995, in AIP Conf. Proc., 348, Unveiling the Cosmic Infrared Background, ed. E. Dwek (New York: AIP), 11

Hauser, M. G. et al. 1998, ApJ, 508, 25

Kashlinsky, A., \& Odenwald, S. 2000, ApJ, 528, 74

Kelsall, T. et al. 1998, ApJ, 508, 44

Lagache, G., Haffner, L. M., Reynolds, R. J., \& Tufte, S. L. 2000, A\&A, 354, 247 
Madau, P., \& Pozzetti, L. 2000, A\&A, 312, L9

Matsumoto, T., Itoh, N., Nakata, K., Cohen, M., \& Tanaka, M. 2000, in preparation

Matsuhara, H., Kawara, K., Sato, Y., Taniguchi, Y. et al. 2000, A\&A, 361, 407

Murakami, H. et al. 1996, PASJ, 48, L41

Noda, M. et al. 1994, ApJ, 428, 363

Noda, M. et al. 1996, SPIE, 2817, 248

Puget, J.-L, et al. 1996, A\&A, 308, L5

Tanihata, C., Takahashi, T., \& Matsumoto, T. 2000, in preparation

Totani, T. et al. 2000 , in this volume

Totani, T., \& Yoshii, Y. 2000, ApJ, 540, 81

Vrtilek, J. M., \& Hauser, M. G. 1995, ApJ, 455, 677

Wright, E. L. 2000, astro-ph/0004192 